enberg FJ, Buchwald H. Risk of osteoporosis in patients treated with long-term intravenous heparir therapy. Curr Surg 1982;39:419.

41. Quyyumi AA, Wright CA, Mockus LJ, Fox M. Mechanisms of nocturnal angina pcctoris: importance of increased myocardi- al oxygen demand in patients with severe coronary artery disease. Lancet 1984;1:1207.

42. Chierchia S, Gallino A, Smith G, Deanfield J, Morgan M, Croom M, Maseri A. Role of heart rate in pathophysiology of chronic stable angina. Lancet 1984;2:1353.

\title{
Reduction in digitalis-associated postinfarction mortality with nadolol in conscious dogs
}

\begin{abstract}
Previously, we have demonstrated an increased incidence of lethal ischemic arrhythmias in postinfarction dogs with clinically observable serum digoxin concentrations, and a significant reduction in digitalis-related lethal ischemic arrhythmias after subacute left stellectomy. In the present study, the protective actions of acute beta-adrenoceptor blockade with nadolol, 1.0 $\mathbf{m g} / \mathbf{k g}$ administered intravenously immediately preceding the induction of posterolateral myocardial ischemia, were assessed in conscious dogs with recent, small anterior myocardial infarctions pretreated with digoxin, $0.0125 \mathrm{mg} / \mathrm{kg} / \mathrm{day}$ intravenously, for 5 to 7 consecutive days (total $n=11$ ). A cohort of postinfarction dogs pretreated with digoxin alone served as a control group (total $n=26$ ). Pre vs postdigoxin electrophysiologic testing indicated reductions in myocardial refractoriness in ventricular noninfarct and infarct zones in both treatment groups, whereas the administration of nadolol tended to reverse the reductions in ventricular refractoriness. Arrhythmia-related deaths in response to posterolateral myocardial ischemia were reduced from 12 of $20(60 \%)$ in the digoxin control group to 2 of $10(20 \%)$ in the digoxin + nadolol group $(p=0.039)$. Serum digoxin concentrations $(1.29 \pm 0.14 \mathrm{ng} / \mathrm{ml}$ vs $1.39 \pm 0.24 \mathrm{ng} / \mathrm{ml})$, underlying anterior myocardial infarct size $(6.9 \pm 1.5 \%$ vs $4.6 \pm 0.9 \%$ of left ventricle), and developing posterolateral myocardial infarct size $(22.8 \pm 2.5 \%$ vs $17.5 \pm 3.6 \%$ of left ventricle) did not differ significantly between the digoxin and digoxin + nadolol groups. Acute beta-adrenoceptor blockade with nadolol appears to reduce digitalis-mediated ischemic postinfarction mortality, possibly because of a salutary increase in ventricular refractoriness. (AM HEART J 1988; 115:67.)
\end{abstract}

Joseph J. Lynch, Ph.D., Jan M. Kitzen, Ph.D., Paul T. Hoff, B.A., and Benedict R. Lucchesi, Ph.D., M.D. Ann Arbor, Mich.

The clinical use of digitalis in the setting of ischemic myocardial injury, including the potential for digitalis to exacerbate ischemic arrhythmias and increase postinfarction mortality, remains controversial. $^{1-7}$ Previous experimental studies, predominantly utilizing acute administrations and toxic doses of digitalis preparations, have demonstrated an enhanced myocardial sensitivity to the arrhyth-

From the Department of Pharmacology, The University of Michigan Medical School.

Supported by National Institutes of Health, Heart, Lung, and Blood Institute grants HL-35325 and HL-05806.

Received for publication May 26, 1987; accepted Aug. 4, 1987.

Reprint requests: Joseph J. Lynch, Ph.D., Department of Pharmacology, M6322 Medical Science Building I, The University of Michigan Medical School, Ann Arbor, MI 48109-0010. mogenic actions of digitalis in the presence of myocardial ischemia, ${ }^{8-10}$ and /or infarction. ${ }^{8,9,11-14}$ A variety of diverse experimental investigations, once again utilizing toxic cardiac glycoside dosing regimens to initiate ventricular arrhythmias, have identified an important interaction between the direct effects of digitalis and the sympathetic nervous system in the genesis of arrhythmias. ${ }^{15-18}$ The aforementioned experimental evaluations taken together suggest that a greater appreciation for and evaluation of the potential interactions between digitalis and the activation of the sympathetic nervous system in the development and treatment of postinfarction arrhythmias may be warranted.

Previously, this laboratory has described the identification of postinfarction dogs at low risk for the 
subsequent development of ischemia-related malignant ventricular arrhythmias via programmed ventricular stimulation testing..$^{19}$ The "low-risk" conscious postinfarction dog model has been used to demonstrate a significantly enhanced susceptibility toward the development of ischemia-related lethal ventricular arrhythmias in the presence of therapeutic serum concentrations of digoxin. ${ }^{20}$ Recently, the interruption of sympathetic neural influences to the left ventricle via the surgical extirpation of the left stellate ganglion 5 to 7 days preceding acute posterolateral ischemia has been shown to be efficacious in reducing the incidence of lethal ventricular arrhythmias in conscious postinfarction dogs with therapeutic serum digoxin concentrations. ${ }^{21}$ In the present study, the cardiac electrophysiologic effects and antiarrhythmic efficacy of acute beta-adrenergic receptor blockade with nadolol are evaluated in conscious postinfarction dogs administered digoxin in a multiday dosing regimen to achieve therapeutic serum concentrations.

\section{METHODS}

Surgical preparation. Detailed methods for the surgical preparation and instrumentation of dogs with anterior myocardial infarction have been described previously. ${ }^{20}$ Briefly, male mongrel dogs (14 to $22 \mathrm{~kg}$ ) were anesthetized with intravenous sodium pentobarbital, $30 \mathrm{mg} / \mathrm{kg}$, and the lungs were ventilated with room air. The left external jugular vein was cannulated for subsequent drug administration. After a left-sided thoracotomy was performed, the left anterior descending coronary artery (LAD) was isolated at the tip of the left atrial appendage, and the left circumflex coronary artery (LCX) was isolated approximately $1 \mathrm{~cm}$ from its origin. Anterior ischemic injury was produced by occlusion of the LAD by means of a snare formed from a loop of silicone rubber tubing passed through a polyethylene cylinder, followed by restoration of LAD blood flow in the presence of a critical stenosis of silk ligature after a period of 2 hours.

During the surgical preparation, an epicardial bipolar electrode ( $1 \mathrm{~mm}$ diameter silver posts, $3 \mathrm{~mm}$ electrode separation) was sutured to the left atrial appendage for pacing. A stainless-steel bipolar plunge electrode $(5 \mathrm{~mm}$ length, $2 \mathrm{~mm}$ electrode separation) was sutured into the interventricular septum adjacent to the right ventricular outflow tract (RVOT) near the site of LAD occlusion for determination of the ventricular excitation threshold, ventricular refractory period, and for programmed ventricular stimulation. Two short ( 2 to $3 \mathrm{~mm}$ ) stainless-steel bipolar electrodes were used for measurement of ventricular activation times and for construction of ventricular strength-interval curves. One bipolar electrode was implanted into the distribution of the LAD immediately distal to the site of coronary occlusion (IZ, infarct zone), whereas the second bipolar electrode was implanted in the LCX distribution (NZ, noninjured, normal zone). Finally, the tip of a silver wire electrode was inserted through the wall and into the lumen of the proximal LCX for subsequent application of electrical current to produce LCX intimal injury and ensuing posterolateral ischemia. Silver disc electrodes were implanted subcutaneously for ECG monitoring, surgical incisions were closed, and the animals were allowed to recover from the surgical anesthetic.

Entry criteria and experimental protocol. Dogs were returned to the laboratory 4 to 5 days after anterior myocardial infarction. Electrophysiologic testing and programmed ventricular stimulation were performed while the animals were conscious and resting comfortably in a sling. Only dogs that were not susceptible to the initiation of either nonsustained or sustained ventricular tachycardia (VT) at baseline programmed stimulation testing were evaluated in the present study. Previous work in this laboratory has demonstrated that postinfarction dogs that are not responsive to initiation of VT at baseline testing are at very "low risk" for development of lethal ventricular arrhythmias in response to the subsequent development of posterolateral ischemia (i.e., ischemia at a site remote from previous myocardial infarction).$^{19}$

Immediately after baseline electrophysiologic and programmed stimulation testing, 11 dogs that were prepared for treatment with digoxin plus acute beta-adrenoceptor blockade with nadolol (DIG + NAD group) began treatment with digoxin $(0.0125 \mathrm{mg} / \mathrm{kg} /$ day intravenously [Lanoxin, Burroughs Wellcome CO, Triangle Park, N.C.], administered in the afternoon as an infusion via the jugular vein) for 5 to 7 consecutive days (mean \pm SEM treatment duration $5.8 \pm 0.5$ days). A previous investigation has demonstrated this dosing regimen to achieve trough digoxin concentrations of approximately 1.0 to 1.6 $\mathrm{ng} / \mathrm{ml}$ after 4 days of treatment. ${ }^{20}$ After the digoxin treatment period, DIG + NAD dogs were subjected to repeat electrophysiologic study and programmed ventricular stimulation in the conscious state. Immediately thereafter, DIG + NAD dogs $(\mathrm{n}=10)$ were given $1.0 \mathrm{mg} /$ $\mathrm{kg}$ nadolol intravenously, followed 30 minutes later by a postnadolol electrophysiologic study and the initiation of electrical current to the LCX intimal surface (see below). It is noteworthly that a previous experimental study has estimated the duration of significant beta-adrenoceptor blockade with a $0.5 \mathrm{mg} / \mathrm{kg}$ intravenous dose of nadolol to be at least 22 hours. ${ }^{22}$ Separate studies in this laboratory determined the duration of a significant attenuation of the chronotropic response to intravenous isoproterenol in conscious dogs treated with $1.0 \mathrm{mg} / \mathrm{kg}$ intravenous nadolol to exceed 24 hours.

In this report, the effect of DIG + NAD treatment on cardiac electrophysiologic parameters and ischemic postinfarction mortality are compared to the results obtained in a cohort of 26 postinfarction control dogs treated with digoxin alone (DIG). This cohort of DIG control animals is comprised of two separate groups of 14 and 12 digoxintreated postinfarction dogs each, which have been described separately in previous reports. ${ }^{20,21}$ The surgical preparation, entry criteria, animal mass $(17.1 \pm 0.4 \mathrm{~kg}$ and $17.8 \pm 0.5 \mathrm{~kg})$, duration of digoxin treatment $(6.2 \pm$ 0.5 and $6.5 \pm 0.3$ days), and resulting serum digoxin 
concentrations $(1.47 \pm 0.19$ and $1.23 \pm 0.14 \mathrm{ng} / \mathrm{ml})$ do not differ between the two groups comprising the DIG control group or among the digoxin controls group and the DIG + NAD group. ${ }^{20,21}$ Repeat electrophysiologic study and programmed ventricular stimulation were performed in all DIG control dogs after digoxin treatment, after which electrical current to the LCX intimal surface was initiated in $20 \mathrm{DIG}$ control dogs (see below). Technical failures associated with the induction of acute posterolateral ischemia (fractured or improperly positioned LCX electrodes; $n=6$ in DIG cohort and $n=1$ in DIG + NAD group) account for the reduction in the number of animals developing pasterolateral ischemia in the DIG and DIG + NAD groups.

Electrophysiologic study and programmed ventricular stimulation. Animals were studied while they were conscious and resting comfortably in a sling. ECG intervals and electrophysiologic parameters were determined immediately before programmed ventricular stimulation. ECG intervals, including a rate-corrected QT interval $\left(Q^{\prime} T_{c}=Q^{\prime} T\right.$ in milliseconds $\left./[\mathrm{K}-\mathrm{R} \text { in seconds }]^{1 / 2}\right)$ were measured in sinus rhythm. A paced QT interval was measured during atrial pacing at $2.5 \mathrm{~Hz}$. RVOT excitation threshold and refractory period, measured during programmed stimulation, and NZ and IZ ventricular activation times were measured during $2.5 \mathrm{~Hz}$ atrial pacing. The RVOT excitation threshold was the minimum voltage required to produce a conducted ventricular impulse $\left(\mathrm{V}_{2}\right)$ by means of a single ventricular extrastimulus $\left(S_{2}\right)$ and a 4 msec pulse duration delivered $300 \mathrm{msec}$ after the $R$ wave of the lead II ECG. The RVOT refractory period was the longest $R-S_{2}$ interval at which a $2 \times$ RVOT threshold voltage stimulus of $4 \mathrm{msec}$ pulse duration failed to elicit a $\mathrm{V}_{2}$ response. $\mathrm{NZ}$ and $\mathrm{IZ}$ activation times, the intervals between the $\mathrm{Q}$ wave of the lead II ECG and the largest deflection of the local ventricular electrogram (Q-EG), were measured on a Tektronix model 5111 storage oscilloscope (Tektronix Inc., Portland, Ore.).

$\mathrm{NZ}$ and IZ ventricular excitation thresholds, relative refractory period (RRP), and effective refractory period (ERP) were determined by the construction of ventricular strength-interval curves according to a modification of the method of Michelson et al., ${ }^{23}$ which has been described in detail previously. ${ }^{20}$ Ventricular strength-interval curves were generated during $2.5 \mathrm{~Hz}$ atrial pacing in the conscious dogs. The strength-interval curves were constructed by the introduction of single ventricular extrastimuli at alternately increasing current strengths and decreasing coupling intervals. During the generation of a curve, current strength for an extrastimulus was increased whenever a reduction in the coupling interval to the preceding complex was sufficient to render the tissue inexcitable. At each site, the excitation threshold was the minimum milliamperage required to elicit a $V_{2}$ response by means of an extrastimulus introduced at a coupling interval of $300 \mathrm{mscc}$ from the preceding complex. The ERP was the longest coupling interval that failed to elicit a $V_{2}$ response at a current strength of $4 \mathrm{~mA}$, whereas the RRP was the longest coupling interval along the strengthinterval curve at which the current required to evoke a $V_{2}$ response increased above the diastolic excitation threshold by more than $0.025 \mathrm{~mA}$ for a $1 \mathrm{msec}$ change in the coupling interval. ${ }^{20,23}$

The pacing protocol for programmed ventricular stimulation was identical to that used by this laboratory in previous investigations. ${ }^{20}$ Briefly, single $\left(\mathrm{S}_{2}\right)$, double $\left(\mathrm{S}_{2} \mathrm{~S}_{3}\right)$, and triple $\left(\mathrm{S}_{2} \mathrm{~S}_{3} \mathrm{~S}_{4}\right)$ premature ventricular extrastimuli (4 msec pulse duration, $2 x$ RVOT threshold voltage) were introduced into the interventicular septum near the RVOT during $2.5 \mathrm{~Hz}$ atrial pacing or sinus rhythm. The basic $\left(S_{1}\right)-S_{2}$ coupling interval was decreased from 350 msec until (RVOT) refractoriness occurred, whereas the $\mathrm{S}_{2}-\mathrm{S}_{3}$ and $\mathrm{S}_{\mathrm{s}}-\mathrm{S}_{4}$ coupling intervals ranged from $182 \mathrm{msec}$ $(5.5 \mathrm{~Hz})$ to $125 \mathrm{msec}(8.0 \mathrm{~Hz})$.

Ischemia at a site remote from previous myocardial infarction: Left circumflex coronary artery intimal stimulation and injury. After the completion of post-treatment programmed ventricular stimulation and electrophysiologic testing, an anodal direct current of $150 \mu \mathrm{A}$ was applied to the intimal surface of the LCX via the previously inserted silver wire electrode. The lead II ECG was recorded directly onto a Grass polygraph or was recorded at preset intervals by a programmable cardiocassette recorder.

After completion of the experiment at 24 hours of electrical stimulation or after the development of lethal arrhythmias, the hearts were excised and thrombus mass within the LCX was determined after removal by careful dissection. The heart was cut into $1 \mathrm{~cm}$ thick transverse sections that were incubated in $0.5 \%$ triphenyltetrazolium chloride in $0.01 \mathrm{~mol} / \mathrm{L}$ phosphate buffer ( $\mathrm{pH} 7.4$ ). Reaction with triphenyltetrazolium forms a red precipitate in viable tissue, whereas infarcted tissue remains pale. ${ }^{24,25}$ Infarct size was quantitated gravimetrically and was expressed as a percentage of total left ventricle.

Determination of serum ion and digoxin concentrations. Blood samples were obtained from all digoxintreated animals immediately before postdigoxin treatment electrophysiologic and programmed stimulation testing. The blood samples were obtained by peripheral venipuncture approximately 16 hours after the preceding administration of digoxin. Serum sodium and potassium concentrations were determined by flame photometry. Serum digoxin concentrations were determined by means of radioimmunoassay technique in the Ligand Assay Laboratory of The University of Michigan Medical School, with the use of sheep antidigoxin antiserum.

Statistical analysis. Data are expressed as mean \pm SEM. Within the DIG control group, pre vs posttreatment comparisons were made by means of a twotailed, paired Student's $t$ test. Within the DIG + NAD group, comparisons among predigoxin, postdigoxin, and postnadolol values were made by means of a repeatedmeasures analysis of variance, followed by Dunnett's test for multiple comparisons. Differences between the DIG and DIG + NAD groups were analyzed by two-tailed, unpaired Student's $t$ test or by Fisher's exact test, when appropriate. For all comparisons, a $p$ value of less than 0.05 was the criterion for statistical significance. 
Table I. Heart rate, ECG intervals, and electrophysiologic parameters for Digoxin control group

\begin{tabular}{|c|c|c|}
\hline Parameter* and Site & Before & After \\
\hline Sinus heart beat (bpm) & $117 \pm 4$ & $113 \pm 5$ \\
\hline PR interval (msec) & $115 \pm 3$ & $117 \pm 3$ \\
\hline QRS interval (msec) & $59 \pm 1$ & $57 \pm 1 \dagger$ \\
\hline $\mathrm{QT}_{\mathrm{c}}$ interval $(\mathrm{msec}) /(\mathrm{sec})^{-1 / 2}$ & $286 \pm 4$ & $265 \pm 4 \ddagger$ \\
\hline QT interval (msec) & $198 \pm 4$ & $187 \pm 2 \S$ \\
\hline \multicolumn{3}{|l|}{ Right ventricular outflow tract } \\
\hline Threshold voltage (V) & $2.0 \pm 0.2$ & $2.4 \pm 0.2$ \\
\hline $\begin{array}{l}\text { Ventricular refractory } \\
\text { period (msec) }\end{array}$ & $158 \pm 3$ & $132 \pm 4 \|$ \\
\hline \multicolumn{3}{|l|}{ Noninfarct zone } \\
\hline Excitation threshold (mA) & $0.37+0.02$ & $0.48+0.07$ \\
\hline $\begin{array}{l}\text { Relative refractory period } \\
\text { (msec) }\end{array}$ & $165 \pm 4$ & $147 \pm 3 \ddagger$ \\
\hline $\begin{array}{l}\text { Effective refractory period } \\
\text { (msec) }\end{array}$ & $135 \pm 5$ & $115 \pm 5 \ddagger$ \\
\hline Activation time (msec) & $15.2 \pm 1.6$ & $16.2 \pm 2.0$ \\
\hline \multicolumn{3}{|l|}{ Infarct zone } \\
\hline Excitation threshold (mA) & $0.47 \pm 0.04$ & $0.69 \pm 0.12 \dagger$ \\
\hline $\begin{array}{l}\text { Relative refractory period } \\
\text { (msec) }\end{array}$ & $177 \pm 5$ & $139 \pm 4 \|$ \\
\hline $\begin{array}{l}\text { Effective refractory period } \\
(\mathrm{msec})\end{array}$ & $150 \pm 5$ & $115 \pm 5 \|$ \\
\hline Activation time (msec) & $19.6 \pm 1.8$ & $17.6 \pm 1.6$ \\
\hline
\end{tabular}

${ }^{*}$ Heart rate, $\mathrm{ECG}$, and noninfarct zone parameters $\mathrm{n}=24$ to 28 ; infarct zone parameters $\mathrm{n}=20$.

$t_{p}<0.05$ compared to "before" value (Student's paired $t$ test). t $p<0.001$ compared to "before" value (Student's paired $t$ test). $\S p<0.01$ compared to "before" value (Student's paired $t$ test). $\| p<0.001$ compared to "before" value (Student's paired $t$ test).

\section{RESULTS}

A total of 37 conscious postinfarction dogs were evaluated in the present study. All animals failed to respond to baseline programmed ventricular stimulation with either nonsustained or sustained VT in accordance with the entry criteria for this study. After baseline electrophysiologic testing, 26 control postinfarction animals $(17.4 \pm 0.3 \mathrm{~kg})$ were administered $0.0125 \mathrm{mg} / \mathrm{kg} /$ day intravenous digoxin for $6.5 \pm 0.3$ consecutive days, followed by repeat electrophysiologic testing and the initiation of acute posterolateral ischemia at a site remote from previous anterior infarction (DIG control group). Eleven postinfarction dogs $(17.5 \pm 0.8 \mathrm{~kg})$ were administered 0.0125 intravenous digoxin for $5.8 \pm 0.5$ consecutive days, followed by postdigoxin electrophysiologic study. Intravenous nadolol, $1.0 \mathrm{mg} / \mathrm{kg}$, was then given to the latter 11 postinfarction dogs, followed by postnadolol electrophysiologic study and the initiation of acute posterolateral ischemia (DIG + NAD group). The responses of the DIG control and DIG + NAD dogs to electrophysiologic testing and to the subsequent development of acute posterolateral ischemia are summarized below.
Response to programmed ventricular stimulation. All DIG control and DIG + NAD postinfarction dogs entered into the evaluation were nonresponsive to baseline programmed stimulation. At the time of postdigoxin repeat programmed ventricular stimulation testing, DIG control dogs had a serum digoxin concentration of $1.33 \pm 0.12 \mathrm{ng} / \mathrm{ml}(\mathrm{n}=26)$, whereas DIG + NAD dogs had a serum digoxin concentration of $1.30 \pm 0.23 \mathrm{ng} / \mathrm{ml}(\mathrm{n}=11)$. Postdigoxin programmed stimulation failed to initiate nonsustained or sustained ventricular tachycardia in 25 of $26 \mathrm{DIG}$ dogs and in 11 of 11 DIG + NAD dogs. One DIG control dog responding to postdigoxin programmed stimulation with rapid VT degenerating into ventricular fibrillation possessed a relatively high serum digoxin concentration $(2.69 \mathrm{ng} / \mathrm{ml})$ and a very large underlying anterior myocardial infarction $(17.9 \%$ of left ventricle). The cumulative anterior myocardial infarct sizes for the two treatment groups at the time of postdigoxin electrophysiologic study and programmed stimulation were (percent of left ventricle): $\mathrm{DIG}+\mathrm{NAD}, 4.7+0.8 \% ;$ DIG $7.5+1.3 \%$; $p=\mathrm{NS})$.

Electrophysiologic responses. ECG and electrophysiologic parameters were determined at baseline testing and after digoxin treatment in DIG control dogs and DIG + NAD dogs, and after the acute intravenous administration of $1.0 \mathrm{mg} / \mathrm{kg}$ nadolol in DIG + NAD dogs. Tables I and II summarize the responses of the DIG and DIG + NAD groups to their treatments, respectively.

The administration of digoxin to the cohort of DIG control dogs was characterized primarily by reductions in both the rate-corrected $\mathrm{QT} \mathrm{T}_{\mathrm{c}}$ and paced QT intervals, and by decreases in refractoriness determined in the normal NZ, "border zone" RVOT, and the IZ of the left ventricle. A previous evaluation of temporally matched, vehicle-treated postinfarction dogs has demonstrated that the observed reductions in ventricular refractoriness are attributable, at least in part, to electrophysiologic changes associated with infarct healing. ${ }^{20}$ In contrast to the findings obtained in vehicle-treated animals, however, the administration of digoxin to postinfarction animals is characterized by a relatively enhanced reduction in both $R R P$ and ERP in the IZ compared to the NZ, such that reductions in the RRP and ERP in the IZ attain a higher degree of statistical significance and no marked differences between the $\mathrm{NZ}$ and IZ are observable for either RRP or ERP after digoxin treatment (Table I).

The ECG and electrophysiologic consequences of digoxin administration to DIG + NAD postinfarction dogs were similar to those observed in the DIG cohort (Table II). The decreases in $\mathrm{QT}_{\mathrm{c}}$ and paced 
Table II. Heart rate, ECG intervals, and electrophysiologic parameters for Digoxin + Nadolol group

\begin{tabular}{|c|c|c|c|}
\hline Parameter* and Site & Before & After digoxin & After nadolol \\
\hline Sinus heart rate (bpm) & $130 \pm 8$ & $115 \pm 9$ & $94 \pm 9 \uparrow$ \\
\hline PR interval (msec) & $109 \pm 3$ & $115 \pm 4$ & $132 \pm 4 \dagger,+$ \\
\hline QRS interval (msec) & $57 \pm 1$ & $58 \pm 1$ & $59 \pm 1$ \\
\hline $\mathrm{QT}_{\mathrm{c}}$ interval $(\mathrm{msec}) /(\mathrm{sec})^{-1 / 2}$ & $293 \pm 7$ & $261 \pm 5 \dagger$ & $257 \pm 8 \dagger$ \\
\hline QT interval (msec) & $192 \pm 6$ & $187 \pm 7$ & $196 \pm 7$ \\
\hline \multicolumn{4}{|l|}{ Right ventricular outflow tract } \\
\hline Threshold voltage (V) & $2.3 \pm 3.2$ & $3.2 \pm 0.2 \dagger$ & 一 \\
\hline Ventricular refractory period (msec) & $155 \pm 7$ & $126 \pm 8 \|$ & - \\
\hline \multicolumn{4}{|l|}{ Noninfarct zone } \\
\hline Excitation threshold (mA) & $0.40 \pm 0.03$ & $0.69 \pm 0.19$ & $0.71 \pm 0.24$ \\
\hline Relative refractory period (msec) & $163 \pm 6$ & $149 \pm 4 \|$ & $157 \pm 5$ \\
\hline Effective refractory period (msec) & $133 \pm 5$ & $116 \pm 7$ & $121 \pm 8$ \\
\hline Activation time (msec) & $5.4 \pm 1.1$ & $7.9 \pm 1.7$ & $9.1 \pm 2.1$ \\
\hline \multicolumn{4}{|l|}{ Infarct zone } \\
\hline Excitation threshold (mA) & $0.81 \pm 0.29$ & $1.1 \pm 0.3$ & $1.1 \pm 0.3$ \\
\hline Relative refractory period (msec) & $171 \pm 9$ & $149 \pm 6 \dagger$ & $159 \pm 6$ \\
\hline Effective refractory period (msec) & $146 \pm 8$ & $114 \pm 8 \dagger$ & $121 \pm 8 \dagger$ \\
\hline Activation time (msec) & $9.7 \pm 2.4$ & $11.4 \pm 1.8$ & $14.2 \pm 2.8$ \\
\hline
\end{tabular}

*Heart rate, ECG, right ventricular outflow tract, and noninfarct zone parameters $\mathrm{n}=11$; infarct zone parameters $\mathrm{n}=10$.

$\dagger p<0.01$ compared to "before" value (Dunnetts test).

$\ddagger p<0.05$ compared to postdigoxin value (Dunnetts test).

$\$ p<0.01$ compared to postdigoxin value (Dunnetts test).

$\| p<0.05$ compared to "hefore" value (Dunnetts test)

Table III. Response to ischemia at a site remote from previous infarction

\begin{tabular}{lcc}
\hline \multicolumn{1}{c}{ Parameter } & Digoxin (n=20) & $\begin{array}{c}\text { Digoxin } \\
(n=10)\end{array}$ \\
\hline Serum digoxin concentration (ng/ml) & $1.29 \pm 0.14$ & $1.39 \pm 0.24$ \\
Duration of digoxin treatment (days) & $6.2 \pm 0.4$ & $5.8 \pm 0.5$ \\
Time to posterolateral ischemia (min) & $352 \pm 40$ & $287 \pm 53$ \\
$\Delta$ Heart rate with ischemia (percentage of preischemic rate) & $25 \pm 5$ & $19 \pm 10$ \\
Myocardial infarct size (percentage of total left ventricle) & $6.9 \pm 1.5$ & $4.6 \pm 0.9$ \\
$\quad$ Anterior infarction & $22.8 \pm 2.5$ & $17.4 \pm 3.6$ \\
Posterolateral infarction & $14 \pm 3$ & $30 \pm 12$ \\
LCX thrombus mass (mg) & $12 / 20(60 \%)$ & $2 / 10(20 \%)^{*}$ \\
24-Hour mortality rate & & \\
\hline
\end{tabular}

${ }^{*} p=0.039$ comparing mortality for digoxin + nadolol group vs digoxin group.

QT intervals, RVOT refractory period, and RRPs and ERPs in the NZ and IZ were quantitatively similar between the DIG + NAD and DIG groups, although the changes observed in the former group achieved a lower degree of statistical significance because of the lower sample size. The subsequent administration of nadolol to digoxin-treated postinfarction dogs resulted in a significant reduction in sinus heart rate and a prolongation in the PR interval. Administration of nadolol also partially reversed the decreases in the RRPs and ERPs of the $\mathrm{NZ}$ and IZ observed after treatment with digoxin (Table II).

Response to ischemia at a site remote from previous myocardial infarction. An anodal current of $150 \mu \mathrm{A}$ was applied to the intimal surface of the proximal
$\mathrm{LCX}$ in $20 \mathrm{DIG}$ and $10 \mathrm{DIG}+$ NAD postinfarction dogs immediately after postdigoxin and postnadolol repeat electrophysiologic studies, respectively. LCX intimal stimulation resulted in intimal injury, thrombus formation, and the ensuing development of posterolateral ischemia in the $20 \mathrm{DIG}$ and 10 DIG + NAD dogs evaluated. Technical failures associated with the placement of the LCX stimulating electrode (see Methods) account for the reduction in the $r$ value for the treatment groups evaluated in the present phase of the study. At the initiation of intimal stimultion of the LCX, the serum digoxin concentrations in the $20 \mathrm{DIG}$ and $10 \mathrm{DIG}+\mathrm{NAD}$ postinfarction dogs were $1.29 \pm 0.14 \mathrm{ng} / \mathrm{ml}$ and $1.39 \pm 0.24 \mathrm{ng} / \mathrm{ml}(p=\mathrm{NS})$, respectively. Serum potassium and sodium concentrations for the 


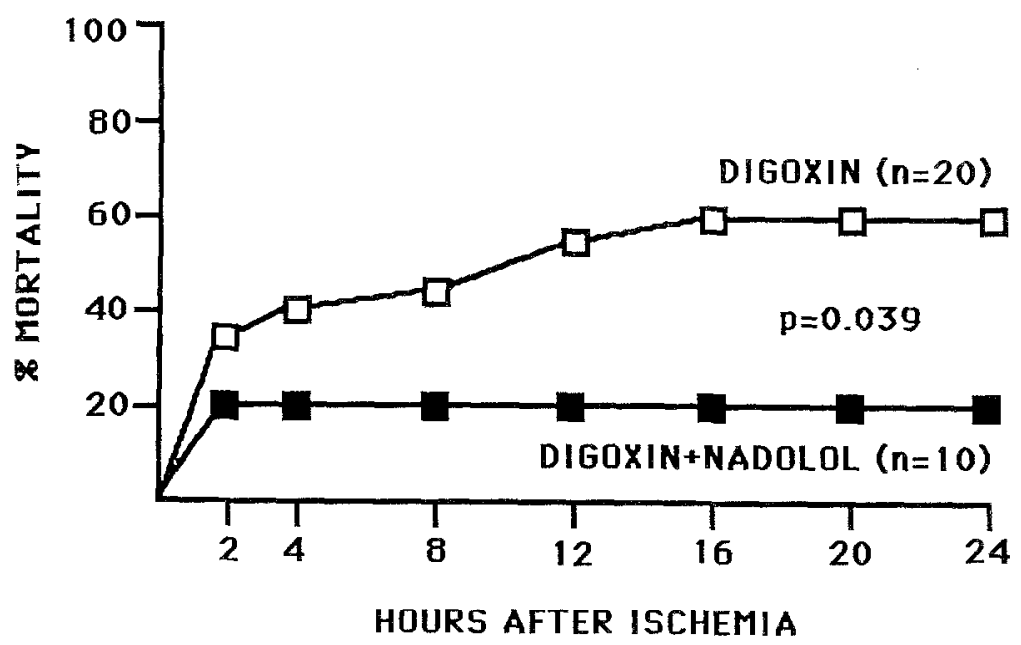

Fig. 1. Mortality rates for digoxin- and digoxin + nadolol-pretreated postinfarction dogs after development of posterolateral myocardial ischemia subsequent to LCX injury and thrombosis.

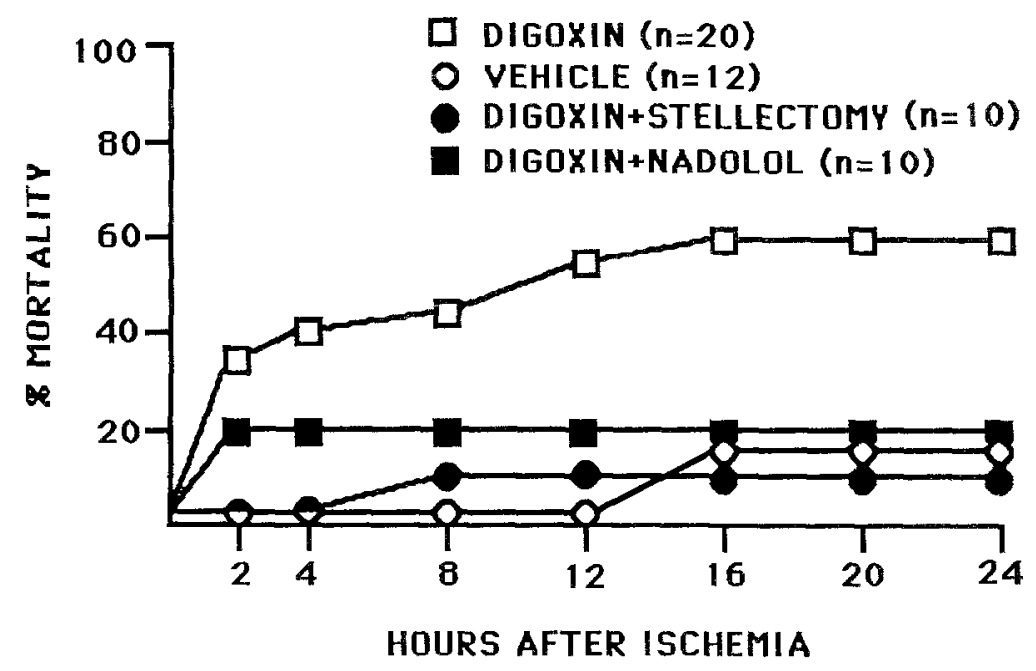

Fig. 2. Cumulative mortality rates for postinfarction dogs pretreated with digoxin, digoxin + nadolol, vehicle, ${ }^{20}$ and digoxin in presence of subacute left stellate ganglion ablation, ${ }^{21}$ in response to development of posterolateral myocardial ischemia and infarction.

$\mathrm{DIG}+\mathrm{NAD}$ dogs were $4.02 \pm 0.02$ and $119.5 \pm 2.8$ $\mathrm{mEq} / \mathrm{L}$, respectively, and are similar to values reported previously for digoxin-treated postinfarction dogs..$^{20,21}$

The responses of DIG and DIG + NAD animals to the development of posterolateral ischemia at a site remote from previous myocardial infarction are summarized in Table III. The durations of LCX intimal stimulation required for ECG manifestation of posterolateral ischemia, the increases in heart rate observed at the onset of posterolateral ischemia, and the sizes of underlying anterior myocardial infarctions did not differ between the DIG and DIG + NAD groups. The 24-hour ischemic postinfarction mortality rate was reduced significantly from 12 of $20(60 \%)$ in the DIG control cohort to 2 of $10(20 \%)$ in the DIG + NAD group $(p=0.039)$. In the DIG group, seven arrhythmia-related deaths were characterized as ventricular fibrillation occurring "suddenly" (i.e., within 60 minutes) after the development of acute posterolateral ischemia, whereas the remaining five DIG deaths were due to ECG-verified ventricular fibrillation occurring during the "delayed" phase of automatic dysrhythmias that developed hours after the onset of acute posterolateral ischemia. The two arrhythmic deaths in the DIG + NAD group were due to ventricular fibrillation developing suddenly after the onset of posterolateral ischemia. Fig. 1 graphically depicts postinfarction mortality as a function of the duration of 
posterolateral ischemia for the DIG and DIG + NAD dogs in the present study. Fig. 2 compares the ischemic postinfarction mortality rates for DIG and DIG + NAD dogs in the present study to the ischemic mortality rates for postinfarction dogs treated with vehicle alone ${ }^{20}$ or with digoxin in the presence of left stellate ganglion ablation. ${ }^{21}$ The reduction in the ischemic postinfarction mortality rate attained in the DIG + NAD group relative to the DIG cohort is similar to those observed in the vehicle-treatment and digoxin + stellectomy groups reported previously. ${ }^{20,21}$

Posterolateral myocardial infarct sizes determined in "delayed" arrhythmic deaths and in 24hour survivors did not differ significantly between the DIG and DIG + NAD groups $(22.3 \pm 2.5$ and $17.4 \pm 3.6 \%$ of left ventricle, respectively), suggesting that the enhanced ischemic postinfarction survival observed in the DIG \pm NAD group was not due to a significant reduction in the severity of the posterolateral ischemic insult. Posterolateral myocardial infarct sizes could not be determined in animals developing "sudden" ventricular fibrillation in response to acute posterolateral ischemia because of the lack of time available for the development of histochemical or morphologic evidence of cell death and tissue necrosis in the ischemic area. ${ }^{24}$ All DIG and DIG + NAD animals surviving 24 hours of ischemia in the LCX distribution developed secondary posterolateral myocardial infarctions and displayed a near $100 \%$ frequency of ventricular ectopic complexes, but were otherwise stable. The mass of thrombus recovered at the site of LCX intimal injury in all dogs who died of arrhythmia and in survivors tended to be greater in DIG + NAD vs DIG animals, probably reflecting the enhanced 24hour survival noted in the DIG + NAD group, allowing for a longer time for thrombus formation (Table III).

\section{DISCUSSION}

Despite their long-standing use in the inotropic support of the failing heart, the administration of cardiac glycosides in the setting of ischemic myocardial injury, and in particular their potential to exacerbate arrhythmias and increase postinfarction mortality, is controversial. ${ }^{1-7}$ It is well established that the direct pharmacologic actions of cardiac glycosides include, in addition to cardiotonic activity, the facilitation of transient inward ionic currents and the formation of delayed afterdepolarizations and "triggered activity"-type arrhythmias, ${ }^{26,27}$ as well as a partially direct coronary vasoconstrictor activity $^{28}$ that might contribute to a worsening of ischemic injury and arrhythmia. Numerous experimental evaluations have demonstrated significant reductions in the intravenous and/or intracoronary lethal ${ }^{8,11}$ and arrhythmogenic ${ }^{9,10,12-14}$ threshold dosages for digitalis preparations during acute myocardial ischemia and after myocardial infarction in vivo. The clinical relevance of the latter findings has been questioned, however, because of the use of acute and rapid administrations of clearly supratherapeutic and toxic concentrations of digitalis preparations in assessing myocardial sensitivity in the setting of ischemic myocardial injury. Recently, Hariman et al. ${ }^{29}$ using microelectrode recording techniques in canine ischemic Purkinje fibers removed from 1-day-old myocardial infarctions, demonstrated an increase in the magnitude of delayed afterdepolarizations and a sustainment of "triggered activity"-type arrhythmias in ischemic preparations exposed to the cardiac glycoside ouabain at a concentration that caused no toxic effect in normal preparations. Lukas and Ferrier, ${ }^{30}$ who also used microelectrode recordings from canine isolated Purkinje fiber preparations, have reported that the "reperfusion" of the preparations after exposure to an "ischemic" buffer solution results in a potentiation of oscillatory afterpotential-induced arrhythmias in the presence of a subthreshold concentration of the cardiac glycoside acetylstrophanthidin. The latter in vitro studies support the concept of an enhancement of myocardial sensitivity toward the arrhythmogenic effects of cardiac glycosides in the setting of ischemic injury.

The findings of numerous experimental studies indicate that many of the cardiovascular effects of the cardiac glycosides, including effects on cardiac conduction and, in part, coronary vasoconstriction, are modulated or affected by the autonomic nervous system. ${ }^{15,18,31-33}$ A variety of experimental observations have been cited as evidence for an interaction between direct drug effects and an activation of the sympathetic nervous system in the genesis of digitalis-induced arrhythmias. ${ }^{15-18,31}$ These observations include: (1) an increase in sympathetic nerve fiber activity to the heart in the presence of arrhythmogenic concentrations of cardiac glycosides ${ }^{34-37}$; (2) an attenuation of digitalis-induced arrhythmias and/or an increase in the arrhythmogenic threshold dosage of digitalis after cardiac denervation ${ }^{33,38-40}$ or spinal cord/central nervous system transections $\mathrm{s}^{31,41-43}$ and (3) a reduction in the electrical threshold for hypothalamic stimulation to cause arrhythmias in the presence of the cardiac glycoside ouabain. ${ }^{44}$ Recently, Hewett and Rosen ${ }^{32}$ evaluated the interaction between the direct electrophysiologic effects of cardiac 
glycosides and adrenergic receptor stimulation in canine isolated Purkinje fibers. In the in vitro cardiac muscle preparations, it was shown that beta-adrenoceptor stimulation enhanced delayed afterdepolarization amplitude and triggered activity in the presence of the cardiac glycoside ouabain, whereas alpha-adrenoceptor agonists and antagonists had no effect. ${ }^{32}$ The latter findings suggest that the arrhythmogenic interaction between cardiac glycosides and sympathetic nervous system activity may be mediated primarily via beta-adrenergic receptor activation. This mechanism may assume enhanced importance in the setting of myocardial ischemia, during which increased neural sympathetic reflex activity ${ }^{45}$ and local catecholamine release $\mathrm{e}^{46,47}$ occur.

Recent investigations in this laboratory have focused on the effect of clinically relevant serum digoxin concentrations, achieved by multiday intravenous dosing regimens, on the frequency and severity of experimental ischemic ventricular arrhythmias, and on developing myocardial infarction. In conscious dogs without previous myocardial infarction, the development of acute posterolateral ischemia in the presence of clinically observable serum digoxin concentrations resulted in the evolution of significantly larger posterolateral myocardial infarctions and the occurrence of postinfarction ventricular ectopic activity that was less sensitive to suppression with conventional antiarrhythmic therapy, as compared to control animals that had not received digoxin pretreatment. ${ }^{48} \mathrm{~A}$ previous report from this laboratory has described the identification of dogs in the subacute phase of anterior myocardial infarction that are at low risk for subsequent development of ischemic ventricular fibrillation via programmed ventricular stimulation testing. ${ }^{19}$ In this "low-risk" postinfarction dog preparation, the administration of digoxin for the achievement of clinically relevant serum concentrations has been associated with a relatively enhanced reduction in ventricular refractoriness in infarcted tissue and a significant increase in the rate of postinfarction arrhythmic mortality occurring in response to posterolateral myocardial ischemia. ${ }^{20}$ Subsequently, Nelson et al..$^{21}$ have demonstrated that an interruption in sympathetic neural input to the ischemically injured left ventricle is effective in reducing the incidence of ischemic lethal ventricular arrhythmias in postinfarction dogs. In the latter study, the surgical extirpation of the left stellate ganglion in "low-risk" digitalized postinfarction dogs 5 to 7 days before the development of acute posterolateral ischemia reduced the incidence of ischemic arrhythmic death from $60 \%$ in shamstellectomized, digitalis-treated animals to $10 \%$ in stellectomized, digitalis-treated animals. ${ }^{21}$ The latter findings suggest that a modulation of the sympathetic nervous system might reduce the ischemic postinfarction mortality rate in the presence of clinically relevant serum digoxin concentrations.

The results of the present investigation support and extend the concept that a modulation of the sympathetic nervous system may be efficacious in reducing the incidence of digitalis-associated ischemic arrhythmic death. In the present study, the acute administration of nadolol immediately before the initiation of LCX stimulation to produce posterolateral ischemia (i.e., nadolol administered approximately 4 to 6 hours preceding posterolateral ischemia) in digoxin-treated postinfarction dogs significantly reduced the incidence of ischemic arrhythmic death relative to digoxin-treated dogs that had not received nadolol. As noted previously, electrophysiologic studies have demonstrated decreases in NZ and IZ ventricular refractoriness with infarct healing and digitalis administration in the conscious postinfarction canine preparation. ${ }^{20,21}$ The administration of digitalis in a therapeutic dosing regimen tends to enhance the decrease in refractoriness preferentially in the IZ. ${ }^{20}$ In general, a decrease in ventricular refractoriness is thought to favor the initiation or perpetuation of reentrant arrhythmias by virtue of the establishment of more stable reentrant circuits with functionally shorter wavelengths. ${ }^{49}$ In contrast, the prolongation of ventricular refractoriness is considered a salutary, potentially antiarrhythmic effect in that a slowing or termination of reentrant arrhythmias may result from a lengthening of the wavelength of a reentrant circuit. ${ }^{49}$ In the present study, the acute administration of nadolol to digitalis-treated postinfarction dogs resulted in a partial reversal of the observed decreases in the ventricular refractoriness of the NZ and IZ. The design of this study does not permit a clear determination of whether the effect of nadolol on ventricular refractoriness was a direct effect of the drug or secondary to a blunting of a digitalisenhanced cardiac sympathetic neural tone. Previous studies have indicated that nadolol possesses minimal direct membrane effects, ${ }^{50,51}$ although a more recent study suggests that it may prolong ventricular refractoriness via a class III electrophysiologic mechanism. ${ }^{52}$ Regardless of the mechanism, an increase in ventricular myocardial refractoriness with nadolol may have conferred, at least in part, some degree of antiarrhythmic protection in this paradigm.

It may not be precluded that the protection 
against ischemic postinfarction mortality observed with nadolol in the present study may have been due to some salutary drug action unrelated to the presence of digitalis. Previous experimental antiarrhythmic evaluations have shown nadolol to be effective in suppressing ischemic ventricular arrhythmias, ${ }^{50,51,53}$ presumably by virtue of its beta-adrenoceptor-blocking activity. Conversely, it has been reported to be relatively ineffective in suppressing existing digoxin-induced arrhythmias in dogs..$^{50}$ It must be kept in mind, however, that the present "low-risk" postinfarction dog model possesses an inherently low rate of ischemic postinfarction death in the absence of digitalis, indicating at least a functional interaction between digoxin and nadolol in this preparation. Although it may be hypothesized that nadolol may have reduced ischemic postinfarction mortality in this study via a lessening in the severity of the ischemic myocardial insult, the lack of a significant difference between the sizes of developing posterolateral myocardial infarctions in "delayed" deaths and survivors in the DIG- and DIG + NAD-treated groups provides indirect evidence for similar degrees of ischemic insult. Finally, the conscious postinfarction dog model used in the present study, in which posterolateral ischemia is produced by electrical injury to the LCX and is followed by the occurrence of sudden lethal arrhythmias in a significant subset of animals, is not amenable to a controlled evaluation of the effect of nadolol treatment on regional myocardial blood flow. However, a salutary effect of nadolol on ischemic blood flow might have been reflected, although not necessarily so, in a reduction in developing posterolateral myocardial infarct size. Regarding the potential effects of beta-adrenoceptor blockade on coronary blood flow in the present investigation, it is noteworthy that previous investigations have determined the neurogenic coronary vasoconstrictor effects of digitalis, in the absence and presence of myocardial ischemia, to be mediated primarily via alpha-adrenoceptor activation. ${ }^{54,55}$ In contrast, pretreatment with the beta-adrenoceptor antagonist propranolol has been shown to protect against the direct vasoconstrictor actions of the cardiac glycoside ouabain and to reduce ensuing arrhythmias and cardiotoxicity in guinea pig isolated perfused hearts..$^{56}$

In summary, acute beta-adrenoceptor blockade with nadolol appears to be effective in reducing ischemic arrhythmic mortality in postinfarction dogs with clinically relevant serum digoxin concentrations. A salutary increase in ventricular myocardial refractoriness may underlie, at least in part, the protective effects of nadolol in this setting. These findings support the concept that a modulation of sympathetic nervous system activity may be effective in reducing digitalis-associated ischemic postinfarction mortality.

\section{REFERENCES}

1. Rahimtoola SH, Gunnar RM. Digitalis in acute myocardial infarction: help or hazard? Ann Intern Med 1975;82:234.

2. Markus FI. Use of digitalis in acute myocardial infarction. Circulation 1980;62:71.

3. Moss AJ, Davis HT, Conard DL, DeCamilla JJ, Odoroff CL. Digitalis-associated cardiac mortality after myocardial in. farction. Circulation 1981;64:1150.

4. Madsen EB, Gilpin E, Henning $\mathrm{H}$, et al. Prognostic importance of digitalis after acute myocardial infarction. J Am Coll Cardiol 1985;3:681.

5. Bigger JT, Fleiss JL, Rolnitzky LM, Merab JP, Ferrick DJ. Effect of digitalis treatment on survival after acute myocardial infarction. Am J Cardiol 1985;55:623.

6. Byington R, Goldstein S, for the BHAT Research Group. Association of digitalis therapy with mortality in survivors of acute myocardial infarction: observations in the Beta-Blocker Heart Attack Trial. J Am Coll Cardiol 1985;6:976.

7. Muller JE, Turi ZG, Stone PH, et al. Digoxin therapy and mortality after myocardial infarction. Experience in the MILIS trial. N Engl J Med 1986;314:265.

8. Bellet S, Johnstone CG, Schecter A. Effect of cardiac infarction on the tolerance of dogs to digitalis. Arch Int Med 1934;54:509.

9. Kumar R, Hood WB, Joisson J, Gilmour DP, Norman JC, Abelman WH. Experimental myocardial infarction. VI. Efficacy and toxicity of digitalis in acute and healing phase in intact conscious dogs. J Clin Invest 1970;49:358.

10. Ku DD, Lucchesi BR. Ischemia-induced alterations in cardiac sensitivity to digitalis. Eur J Pharmacol 1979;57:135.

11. Travell J, Gold H, Modell W. The effect of experimental cardiac infarction on response to digitalis. Arch Int Med 1983;61:184.

12. Hood WB, McCarthy B, Lown B. Myocardial infarction following coronary ligation in dogs. Hemodynamic effects of isoproterenol and acetylstrophanthidin. Circ Res 1967; 21:191.

13. Morris JJ, Taft CV, Whalen RE, McIntosh HD. Digitalis and experimental myocardial infarction. AM HEART $J$ 1969; $77: 342$

14. Iesaka J, Aonuma $\mathrm{K}$, Gosselin AJ, et al. Susceptibility of infarcted canine hearts to digitalis-toxic ventricular tachycardia. J Am Coll Cardiol 1983;2:45.

15. Gillis RA, Pearle DL, Levitt B. Digitalis: a neuroexcitatory drug. Circulation 1975;52:739.

16. Roberts J, Kelliher GJ, Lathers CM. Role of adrenergic influences in digitalis-induced ventricular arrhythmias. Life Sci 1976;18:665.

17. Lathers CM, Roberts J. Digitalis cardiotoxicity revisited. Life Sci 1980;27:1713.

18. Gillis RA, Quest JA. The role of the nervous system in the cardiovascular effects of digitalis. Pharmacol Rev 1980; $31: 19$.

19. Wilber DJ, Lynch JJ, Montgomery DG, Lucchesi BR. Postinfarction sudden death: significance of inducible ventricular tachycardia and infarct size in a conscious canine model. AM HEART J 1985;109:8.

20. Lynch JJ, Montgomery DG, Lucchesi, BR. Facilitation of lethal ventricular arrhythmias by therapeutic digoxin in conscious postinfarction dogs. AM HEART J 1986;111:883.

21. Nelson SD, Lynch JJ, MacEwen S, Lucchesi BR. Antifibrillatory actions of left stellectomy in digitalis-mediated malignant ventricular arrhythmias. Circulation 1986;74:II-30. 
22. Lee RJ, Evans DB, Baky SH, Laffan RJ. Pharmacology of nadolol (SQ11725), a beta-adrenergic antagonist lacking direct myocardial depression. Eur J Pharmacol 1975;33:371.

23. Michelson EL, Spear JF, Moore EN. Electrophysiologic and anatomic correlates of sustained ventricular tachyarrhythmias in a model of chronic myocardial infarction. Am J Cardiol 1980;45:583.

24. Fishbein MC, Meerbaum S, Rit J, et al. Early phase acute myocardial infarct size quantitation: validation of the triphenyltetrazolium chloride tissue enzyme staining technique. AM HeART J 1981;101:593.

25. Vivaldi MT, Kloner RA, Schoen FJ. Triphenyltetrazolium staining of irreversible ischemic injury following foronary artery occlusion in rats. Am J Pathol 1985;121:522.

26. Cranefield PF. Action potentials, afterpotentials and arrhythmias. Circ Res 1977;41:415.

27. Ferrier GR. Digitalis arrhythmias: role of oscillatory afterpotentials. Prog Cardiovasc Dis 1977;19:459.

28. Tanz RD. Possible contribution of digitalis-induced coronary constriction to toxicity. AM HEART J 1986;111:812.

29. Hariman RJ, Zeiler RH, Gough WB, El-Sherif N. Enhancement of triggered activity in ischemic Purkinje fibers by ouabain: a mechanism of increased susceptibility to digitalis toxicity in myocardial infarction. J Am Coll Cardiol 1985; $5: 672$.

30. Lukas A, Ferrier GR. Interaction of ischemia and reperfusion with subtoxic concentrations of acetylstrophanthidin in isolated cardiac ventricular tissues: effects on mechanisms of arrhythmia. J Mol Cell Cardiol 1986;18:1143.

31. Somberg JC. Digitalis: neurally-mediated arrhythmogenic and coronary vasoconstrictor properties. J Clin Pharmacol 1985;25:529.

32. Hewett KW, Rosen MR. Alpha and beta adrenergic interac tions with ouabain-induced delayed afterdepolarizations. J Pharmacol Exp Ther 1984;229:188.

33. Kujime K, Natelson BH. Effects of stellectomy on cardiac rhythm disturbances induced by ouabain in guinea pigs. $J$ Pharmacol Exp Ther 1984;229:113.

34. Gillis RA, Raines A, Sohn YJ, Levitt B, Standaert FG. Neuroexcitatory effects of digitalis and their role in the development of cardiac arrhythmias. J Pharmacol Exp Ther 1972;183:154.

35. Pace DG, Gillis RA. Neuroexcitatory effects of digoxin in the cat. J Pharmacol Exp Ther 1976;199:583.

36. Weaver LC, Akerat, Brody TM. Digoxin toxicity: primary sites of drug action on the sympathetic nervous system. J Pharmacol Exp Ther 1976;197:1.

37. Lathers CM, Kelliher GJ, Roberts J, Beasley AB. Nonuniform cardiac sympathetic discharge. Mechanism for coronary occlusion and digitalis-induced arrhythmia. Circulation 1978;57:1058.

38. Erlij D, Mendez R. The modification of digitalis intoxication by excluding adrenergic influences on the heart. $J$ Pharmacol Exp Ther 1964;144:97.

39. Boyajy LC, Nash CB. Alteraiton of ouabain toxicity by cardiac denervation. Toxicol Appl Pharmacol 1966;9:199.

40. Lathers CM, Gerard-Ciminera JL, Baskin SI, et al. Role of the adrenergic nerve terminal in the digitalis-induced cardiac toxicity: a study of the effects of pharmacological and surgical denervation. J Cardiovasc Pharmacol 1982;4:91.

41. Cagin NA, Somberg J, Bounous H, Mittag T, Raines A, Levitt $B$. The influence of spinal cord transection on the capacity of digitoxin to induce cardiotoxicity. Arch Int Pharmacodyn Ther 1974;207:340.

42. Helke CJ, Zavadil AP, Gillis RA. Forebrain noradrenergic mechanisms and digitalis-induced ventricular arrhythmias. $J$ Pharmacol Exp Ther 1979;208:57.

43. Somberg JC, Smith TW. Localization of the neurallymediated arrhythmogenic properties of digitalis. Science 1979:204:321.

44. Evans DE, Gills RA. Effects of ouabain and its interaction with diphenylhydantoin on cardiac arrhythmias induced by hypothalmic stimulation. J Pharmacol Exp Ther 1975; 195:577.

45. Malliani A, Lombardi F. Neural reflexes associated with myocardial ischemia. In: Schwartz PJ, Brown AM, eds. Neural mechanisms in cardiac arrhythmias. New York: Raven Press, 1978:209.

46. Muntz KH, Hagler HK, Boulas J, Willerson JT, Buja LM. Redistribution of catecholamines in the ischemic zone of the dog heart. Am J Pathol 1984;114:64.

47. Schomig A, Dart AM, Dietz R, Kubler W, Mayer E. Paradoxical role of neuronal uptake for the locally mediated release of endogenous noradrenalin in the ischemic myocardium. J Cardiovasc Pharmacol 1985;7(suppl 5):40.

48. Lynch JJ, Lucchesi BR. Effect of digoxin on the extent of injury and the severity of arrhythmias during acute myocardial ischemia and infarction in the dog. J Cardiovasc Pharmacol 1987. (In press.)

49. Wellens HJJ, Brugada P, Farre J. Ventricular arrhythmias: mechanisms and actions of antiarrhythmic drugs. AM HEART J 1984;107:1053.

50. Evans DB, Peschka MT, Lee RJ, Laffan RJ. Antiarrhythmic action of nadolol, a beta-adrenergic receptor blocking agent. Eur J Pharmacol 1976;35:17.

51. Patterson E, Lucchesi BR. Antifibrillatory actions of nadolol. J Pharmacol Exp Ther 1982;223:144.

52. Taggart P, Donaldson R, Abed J, Nashat F. Class III action of beta-blocking agents. Cardiovasc Res 1984;18:683.

53. Patterson E, Scherlag BJ, Lazzara R. Mechanism of prevention of sudden death by nadolol: differential actions on arrhythmia triggers and substrate after myocardial infarction in the dog. J Am Coll Cardiol 1986;8:1365.

54. Hamlin NP, Willerson JT, Garan H, Powell WJ. The neurogenic vasoconstrictor effect of digitalis on coronary vascular resistance. J Clin Invest 1974;53:288.

55. Sagar KB, Hanson EC, Powell WJ. Neurogenic coronary vasoconstrictor effects of digitalis during acute global ischemia in dogs. $J$ Clin Invest 1977;60:1248.

56. Tanz RD, Russel NJ, Banerian SP, Sharp VH. Ouabaininduced tachyarrhythmias and cell damage in isolated perfused guinea-pig hearts. Protection by propranolol. J Mol Cell Cardiol 1982;14:655. 